\title{
Le savoir turc à la conquête du Moyen-Orient : sociologie d'un champ d'expertise de politique étrangère
}

\section{Turkish Academia and the Middle East: Sociology of a Foreign Policy Experts' Field}

par Jean-Baptiste Le Moulec

Depuis le premier blocage, dès 2005, des négociations d'adhésion de la Turquie à l'Union européenne et l'intensification des initiatives turques en direction du Proche-Orient ${ }^{1}$ qui en ont découlé, universitaires et analystes de tous bords, en Turquie comme ailleurs, ont largement commenté la «nouvelle» politique étrangère turque. Plus précisément, entre 2006 et 2013, l'identité islamique et conservatrice du gouvernement AKP (Adalat ve Kalkınma Partisi, Parti de la justice et du développement) a souvent été avancée pour expliquer l'orientation de ses relations avec le Moyen-Orient ${ }^{2}$.

Je propose une analyse sociologique des liens entre une partie du champ académique turc engagé dans le développement d'une expertise de la politique moyen-orientale du gouvernement et les institutions de l'État en charge de la politique extérieure comme de la recherche scientifique. Sociologie des relations savoir-pouvoir, cette étude s'appuie sur une soixantaine d'entretiens conduits à Ankara et à Istanbul entre octobre 2012 et mars $2016^{3}$ ainsi que sur le dépouillement d'un vaste corpus de revues académiques ou de vulgarisation et d'articles de presse, tous publiés en Turquie entre 1995 et $2016^{4}$. La production académique et plus largement experte sur le Moyen-Orient s'est intensifiée au cours des années 2000 en Turquie. Dominée par une orientation géopolitique, voire géostratégique, elle laisse souvent de côté l'étude ethnographique fondée sur des séjours de terrain et sur la maîtrise des langues locales (arabe, persan, hébreu).

\footnotetext{
${ }^{1}$ Parmi les plus significatives : la consolidation de l'amitié turco-syrienne à partir de 1999 ; un accord de libre-échange en 2004 avec ce pays, le soutien politique et humanitaire à la cause palestinienne et la quasi-rupture des relations avec Israël entre 2008 et 2015 ; les tournées triomphales d'Erdoğan et de Davutoğlu à la fin des années 2000 au Proche-Orient et en Afrique du Nord, la multiplication des contrats de BTP turcs (notamment en Libye, en Algérie, au Maroc) et la hausse des exportations turques vers cette région grâce à l'appui du personnel politique et diplomatique turc. Baskın Oran (dir.), Türk Dış Politikası: Kurtuluş Savaşindan Bugüne (La politique étrangère turque : de la Guerre d'indépendance à nos jours), vol. 3, 2001 - 2012, Istanbul, İletişim, 2013.

2 Bülent Aras, "The Davutoğlu Era in Turkish Foreign Policy», Insight Turkey, 11 (3), 2009, p. 127- 142 ; Burhanettin Duran, Nuh Y1lmaz, « Islam, Models and the Middle East: The New Balance of Power Following the Arab Spring », Perceptions, 18 (4), 2013, p. 139- 170.

${ }^{3}$ Les entretiens, réalisés la plupart du temps en turc, parfois en arabe, ont été traduits par mes soins.

${ }^{4}$ Cette analyse reprend plusieurs éléments présentés plus longuement dans Jean-Baptiste Le Moulec, «Janissaires du savoir. Sociologie des producteurs et diffuseurs de savoirs sur le Moyen-Orient en Turquie (1998-2015) », thèse de doctorat en science politique, Institut d'études politiques d'Aix-enProvence, 2016.
} 
Expliquer ce biais disciplinaire implique avant tout d'aller à la rencontre des auteurs en adoptant une approche assez largement biographique.

Le sursaut éditorial dédié à la «région » (bölge) et l'ensemble de la dynamique experte turque vers le Moyen-Orient - Ortadoğu - dans les années 2000 et 2010 sont étroitement liés aux initiatives du gouvernement AKP durant cette même période. Il convient cependant de se pencher en premier lieu sur les enjeux de la définition pour le moins mouvante de cet Ortagdoğu. De fait, la définition du Moyen-Orient varie selon les affinités idéologiques des auteurs. Il peut notamment désigner une zone géographique dont le centre est la Turquie et dont la périphérie inclut aussi bien les Balkans que le Caucase, le bassin de la mer Noire et le Proche-Orient ${ }^{5}$. Le tournant doctrinal qui s'est opéré au début des années 2000 dans la politique étrangère de la Turquie a consisté pour celle-ci à ne plus s'identifier exclusivement au rôle de pivot régional, périphérique, établi par les stratèges de l'OTAN, mais à incarner une centralité définissant ses propres priorités vis-à-vis de sa périphérie immédiate. Corrélativement, les objectifs de la Turquie au «Moyen-Orient »dans les années 2000 ont été déterminés de manière concentrique, les pays limitrophes - Syrie, Irak, Iran - bénéficiant, au moins jusqu'en 2010, d'une attention accrue par rapport aux pays plus éloignés. Au paramètre de la distance s'est superposée une lecture civilisationnelle qui, se référant aux dernières décennies de l'Empire Ottoman, a amené le gouvernement turc à porter assistance à une population symboliquement importante: les Palestiniens de Palestine et les réfugiés palestiniens de Jordanie et du Liban. En d'autres termes, le Moyen-Orient de la Turquie de la première décennie AKP a été avant tout le Proche-Orient arabe. Il a pris une coloration nord-africaine à partir de la «Révolution de jasmin » (Tunisie, 2010) et de l'insurrection libyenne (2011). La doctrine géostratégique de l'AKP n'a pas remis en cause le terme de Moyen-Orient, pourtant forgé aux États-Unis au début du XX $X^{\mathrm{e}}$ siècle, mais elle en a modifié le contenu au gré de ses orientations.

Je m'attache ici à décrire et à qualifier la relation à la fois dense, diffuse et non dépourvue d'ambigüités entre producteurs turcs de savoirs en lien avec le Moyen-Orient et acteurs diplomatiques et politiques. Évoquer une affinité idéologique pour expliquer que les seconds soient devenus les bailleurs de fonds des premiers aurait constitué un excès de simplification. Des liens réticulaires multiples et complexes ont existé dans ce qui, au début des années 2010, alors que survenaient les insurrections arabes, s'apparente à un sous-champ ${ }^{6}$ des sciences

\footnotetext{
${ }^{5}$ Yael Navaro-Yashin, Faces of the State, Princeton, Princeton University Press, 2002.

${ }^{6}$ Le choix du terme «champ » renvoie à la sociologie de Pierre Bourdieu quand bien même c'est l'autonomie même de cette entité qui est en question ici. Il m'a néanmoins paru plus adéquat que le terme « nébuleuse » (Christian Topalov (dir.), Laboratoires du nouveau siècle. La nébuleuse réformatrice et ses réseaux en France 1880-1914, Paris, Éditions de 1'EHESS, 1999), connoté négativement et moins axé sur
} 
sociales turques, disciplines plus largement considérées comme camérales ${ }^{7}$ dans la tradition universitaire turque ${ }^{8}$. Il convient donc de recourir ici à un arsenal conceptuel relevant à la fois de la sociologie de l'action publique et de la sociologie des sciences, ce qui n'a été que peu pratiqué sur le cas turc à la différence d'autres terrains ${ }^{9}$.

Dès lors, je m'appuierai sur la sociologie de l'expertise et des think tanks ${ }^{10}$ puisque ces structures apparaissent d'emblée dans le domaine étudié. Toutefois, souhaitant préciser leur rôle dans un cadre politique plus large, je mobiliserai les notions de policy communities $^{11}$, de policy networks ${ }^{12}$ et plus encore d'epistemic community tant celle-ci semble prometteuse pour décrire la formation d'un groupe spécifique d'intellectuels organiques dans l'espace qui m'intéresse. À partir de la réconciliation turco-syrienne de 1999, et grâce à un soutien croissant des bailleurs de fonds publics et privés turcs et internationaux, un champ professionnel s'est constitué en Turquie dans l'interstice des administrations et agences d'État ${ }^{13}$, des universités, des médias et des organisations culturelles et/ou humanitaires non gouvernementales.

Revenir sur les conditions historiques de la production de savoirs sur le Moyen-Orient arabe en Turquie, et notamment sur son antériorité à l'ère AKP, permettra ensuite de souligner la

les positions des acteurs. En revanche, le terme « constellation » convient bien pour la période pré-AKP, marquée par un fort éparpillement des acteurs individuels et une faible institutionnalisation du champ.

${ }^{7}$ Pierre Favre, Naissance de la science politique en France (1870-1914), Paris, Fayard, 1989.

${ }^{8}$ Erozan Boğaç, Ilter Turan, «The Development of Political Science in Turkey », Political Science and Politics, 37 (2), 2004, p. 359- 363.

${ }^{9}$ Par exemple, Hocine Khelfaoui, «Le champ universitaire algérien entre pouvoirs politiques et champ économique », Actes de la recherche en sciences sociales, 148 (3), 2003, p. 34- 46 ; Mostefa Lacheraf, «Les intellectuels, le politique et le pouvoir : généralités et cas spécifiques dans les pays du Tiers Monde et en Algérie », dans M. Lacheraf, Algérie et Tiers Monde : agressions, résistances et solidarités intercontinentales, Alger, Bouchène, 1989; Thomas Medvetz, «Les think tanks aux États-Unis : l'émergence d'un sous-espace de production des savoirs », Actes de la recherche en sciences sociales, 176- 77 (1-2), 2009, p. 82- 93.

${ }^{10}$ CRESAL (Centre de recherches et d'études sociologiques appliquées de la Loire), «Situation d'expertise et socialisation des savoirs ", Université de Saint-Étienne, 1985. Les Actes de cette table ronde sont disponibles sur http://science-societe.fr/situation-d\%E2\%80\%99expertise-et-socialisation-dessavoirs-cresal-saint-etienne-1985/; Arnaud Le Gall, «L'État et les think tanks internationalistes. La structuration d'un espace de production d'expertise des années 1960 à nos jours », dans Olivier Ihl, Yves Deloye, Alfredo Joignant (dir.), Gouverner par la science. Perspectives comparées, Grenoble, Presses universitaires de Grenoble, 2013 ; Lisa Stampnitzky, « Experts, États et théorie des champs. Sociologie de l'expertise en matière de terrorisme », Critique internationale, 59, 2013, p. 89- 104.

${ }^{11}$ Andrew Jordan, Jeremy Richardson, «Policy Communities: The British and European Policy Style », Policy Studies Journal, 11 (4), 1983, p. 603- 615 ; Peter M. Haas, Knowledge, Power, and International Policy Coordination, Los Angeles, University of South Carolina Press, 1997.

${ }^{12} \mathrm{C}$ 'est à regret que j'emploie ici l'expression anglaise mais sa version française, « réseau de politique publique », pose problème du fait de l'interrogation sur le caractère public d'une politique étrangère.

${ }^{13}$ Principalement l'administration du ministère des Affaires étrangères, du bureau du Premier ministre, du Conseil turc pour la recherche scientifique et technologique (Türk Bilimsel ve Teknik Araştırma Kurulu, TÜBITAK) et du Conseil de l'enseignement supérieur (Yüksek Öğretim Kurulu, YÖK, équivalent du CNU français). 
spécificité de la configuration experte qui apparaît sous l'AKP sur la base d'une typologie des trajectoires individuelles, en particulier du groupe moteur du dispositif, la communauté épistémique réunie autour d'Ahmet Davutoğlu.

Un domaine d'expertise ancien en Turquie mais qui a longtemps pâti d'un manque de légitimité politique

En dépit de l'acharnement des intellectuels organiques de $1^{\prime} \mathrm{AKP}^{14}$ à promouvoir le récit qui fait de ce parti le réconciliateur des Turcs et des Arabes, l'intérêt de certains courants intellectuels turcs pour le Moyen-Orient arabe, prémices d'une expertise au service de la politique étrangère, a nettement précédé l'apparition du parti d'Erdoğan et n'a pas toujours relevé de la mouvance conservatrice islamique.

Dès les premières années de la République, durant les années 1920, alors que l'enseignement religieux passait sous le contrôle de l'État, des facultés de théologie arabes comme Al-Azhar (Le Caire), Umm Al-Qura (La Mecque) ou celle de l'Université de Damas ont attiré des étudiants $\operatorname{turcs}^{15}$. Cette tendance s'est poursuivie avec une densité variable durant tout le $\mathrm{XX}^{\mathrm{e}}$ siècle. Par ailleurs, à partir du milieu des années 1960 et durant les années 1970, les militants communistes maoïstes turcs ont noué des liens étroits avec leurs homologues palestiniens, libanais, syriens et kurdes. Rejoindre alors les rangs de l'OLP au Liban était autant un exil pour fuir la répression croissante de l'appareil d'État et des milices d'extrême droite ${ }^{16}$ qu'un rite initiatique dans la lutte anti-impérialiste ${ }^{17}$ de certaines franges de la jeunesse turque. Les réseaux confessionnels ou politiques tissés au cours de ces années ainsi que la connaissance empirique de certaines parties du Proche-Orient ont été mis à profit dès les années 1980 lorsque certains de ces militants,

\footnotetext{
${ }^{14}$ L'expression « intellectuel organique » renvoie à Antonio Gramsci, Guerre de mouvement et guerre de position, Paris, La Fabrique Éditions, 2012. Elle est reprise par Gisèle Sapiro, "Modèles d'intervention politique des intellectuels : le cas français », Actes de la recherche en sciences sociales, 176- 77 (1-2), 2009 , p. 8- 31.

${ }^{15}$ En marge des entretiens, j'ai réalisé une étude prosopographique retraçant le parcours de 143 personnalités qui se sont signalées par leur production de savoir ou leur participation au débat médiatique turc relatif au Moyen-Orient et dont $10 \%$, pourcentage faible mais non négligeable, avaient effectué une partie de leur cursus universitaire dans une faculté de théologie arabe.

${ }^{16}$ Benjamin Gourisse, «Participation électorale, pénétration de l'État et violence armée dans la crise politique turque de la seconde moitié des années 1970. Contribution à l'analyse des crises politiques longues », Politix, 98 (2), 2012, p. 171- 193.

${ }^{17}$ Feyizoğlu Turhan, Denizler ve Filistin (Les « Deniz » et la Palestine), Istanbul, Alfa, 2011.
} 
assagis et revenus en Turquie, ont intégré la presse, l'université et/ou l'entourage des décideurs politiques $^{18}$.

Parallèlement, au cours des années 1980, la montée en puissance du mouvement autonomiste kurde fédéré par le PKK (Partiya Karkerên Kurdistan, Parti des travailleurs du Kurdistan) d'Abdullah Öcalan a fini par déboucher sur un conflit ouvert avec les forces de sécurité turques. L'aggravation du conflit dans les années 1990 et son internationalisation du fait de l'implantation transnationale du PKK ont alors fait du développement d'une expertise turque au service de la politique sécuritaire et extérieure nationale un impératif. C'est dans ce contexte, par exemple, que l'ancien militant maoïste et supplétif turc de l'OLP, Cengiz Çandar, devenu journaliste vers la fin des années 1970, a collaboré avec l'administration du Premier ministre puis président Turgut Özal, devenant ainsi le modèle type de conseiller du prince ${ }^{19}$. D'autres, comme les journalistes Faik Bulut et Bereket Kâr, ont suivi une trajectoire similaire bien que moins prestigieuse. À partir de la réconciliation turco-syrienne de 1999, tous ces «spécialistes ès Kurdes » se sont mués en spécialistes du Moyen-Orient. À ce titre, ils ont été sollicités tant par les médias que par les structures de recherche, ce qui est révélateur non seulement de la position des Kurdes et de la «question kurde » dans l'imaginaire national turc, mais aussi de la carence d'expertise turque sur le monde arabe à la fin des années 1990. La question kurde a fait en effet office de thème de passage, de sas, vers le Proche-Orient arabe de la même façon que certains intellectuels kurdes internationalisés par leurs études ou leur carrière ont fait office d'experts de l'ensemble du Moyen-Orient ${ }^{20}$. Ainsi, dès la fin des années 1990, Cengiz Çandar a été considéré dans les médias, les universités ou les couloirs du ministère des Affaires étrangères comme le meilleur spécialiste turc du monde arabe. Il ne s'agit pas de relativiser la pertinence des savoirs dont il était, avec d'autres, le dépositaire, mais de souligner la facilité avec laquelle ces journalistes et/ou intellectuels ont occupé l'espace nouvellement créé de l'expertise turque sur le Moyen-Orient. En fait, ces premiers experts tenaient moins leur statut de titres universitaires ou de positions professionnelles que d'interactions directes plus ou moins récentes avec les sociétés du Proche-Orient. Dès les années 1990, ce crédit empirique a suscité l'intérêt du champ universitaire turc où, par tradition, le savoir en sciences sociales sur l'étranger tirait moins sa légitimité d'un rapport direct aux sources primaires et au terrain que de la maîtrise des connaissances théoriques de la discipline, quitte à n'exploiter que des sources secondaires.

${ }^{18}$ Cengiz Çandar, Mezopotamya Ekspresi. Bir Tarih Yolculuğu (Mésopotamie Express. Un voyage d'histoire), Istanbul, İlestişim, 2012.

${ }^{19}$ Ibid.

${ }^{20}$ Yohanan Benhaim, «Quelle politique kurde pour l'AKP ? », Politique étrangère, 2, 2014, p. 39-50. 
La dernière composante ayant conduit à la constitution d'un champ expert autour de la politique moyen-orientale de l'AKP a consisté en trois initiatives de mobilisation intellectuelle rassemblant les mêmes acteurs. La première est celle de la Plateforme Abant (Abant Platformu), lancée en 1998 par la Fondation des journalistes et écrivains (Gazeteciler ve Yazarlar Vakfi) à Bolu (Anatolie centrale). La Fondation avait été créée par des journalistes proches de la néoconfrérie de Fethullah Gülen mais la Plateforme Abant a rassemblé des intellectuels de tous les horizons idéologiques et confessionnels lors de grandes conférences qui ont lieu entre 1998 et $2016^{21}$. Les sujets abordés lors de ces réunions concernaient aussi bien la société turque que le rapport au monde des Turcs. C'était un cénacle où anciens «gauchistes » et «islamistes » (solcular ve islamcilar) dialoguaient sur l'avenir de la Turquie au-delà des clivages politiques et sociaux traditionnels. La deuxième mobilisation est celle de la Conférence moyen-orientale (Ortadoğu Konferans1), lancée par quelques intellectuels turcs et kurdes au passé marxisant, qui a consisté, entre 1998 et 2003 environ, à se rendre dans les capitales du Proche-Orient pour rencontrer des intellectuels arabes, persans et israéliens ${ }^{22}$. La troisième est celle d'un collectif féminin, Femmes du Moyen-Orient (Ortadoğu Kadınları), qui a organisé également, au début des années 2000, des rencontres avec des actrices de la vie intellectuelle et politique des pays arabes, d'Iran et d'Israël. Il rassemblait des universitaires et journalistes turques ${ }^{23}$ qui partaient en délégation tantôt vers Téhéran, tantôt vers Dubaï ou Damas. Des invitations étaient également adressées à certaines figures féminines moyen-orientales. Il semble toutefois que, faute de moyens financiers ${ }^{24}$, ces voyages et invitations n'aient pu se pérenniser au-delà de 2003.

Ces trois types de mobilisation intellectuelle relativement informelle ont perdu en substance vers le milieu des années 2000, lorsque le temps du «dialogue citoyen » a été révolu et qu'une politique d'État portée par le gouvernement AKP est venue relayer l'intérêt surtout journalistique pour le Moyen-Orient. Dès lors, l'expertise académique - incluant les think tanks pour lesquels les attaches avec le champ universitaire sont une ressource indispensable - a pris le pas sur le débat entre intellectuels critiques issus de courants politiques divers. En somme, après une phase

\footnotetext{
${ }^{21}$ Entretien avec Kerim Balcı, rédacteur en chef, Turkish Review (Groupe de presse Zaman Grubu), Istanbul, février 2013 ; entretien avec Veysel Ayhan, président du think tank International Middle East Peace Research Center, IMPR, Ankara, avril 2013.

${ }^{22}$ Entretien avec Mete Çubukçu, journaliste et présentateur d'une émission de politique internationale (chaîne NTV), Istanbul, novembre 2012 ; entretien avec Nuray Mert, maitresse de conférences en histoire (Université d'Istanbul-faculté de sciences politiques), Istanbul, novembre 2012.

${ }^{23}$ Entretien avec Fulya Atacan, professeure de sociologie (Université Y1ldız Teknik Üniversitesi-faculté de sciences politiques), Istanbul, octobre 2012 ; entretien avec N. Mert, cité.

${ }^{24} \mathrm{~N}$. Mert m'a ainsi raconté qu'elle payait elle-même ses voyages mais que certaines de ses collègues bénéficiaient de ressources institutionnelles. Cette universitaire qui a également une intense activité en tant que chroniqueuse de presse - köşe yazarı - a participé aux trois initiatives.
} 
de construction d'un consensus intellectuel sur la nécessité d'une implication turque gouvernementale et transnationale au Moyen-Orient arabe et persan, ces intellectuels se sont tournés vers une production de savoirs essentiellement académiques.

Les think tanks turcs entre renouvellement chronique et permanences systémiques

Le gouvernement AKP est arrivé au pouvoir en novembre 2002 avec un programme européen et un calendrier de réformes institutionnelles. Ce n'est qu'à la fin de l'année 2015, lorsque les négociations d'adhésion ont rencontré des difficultés, que Recep Tayyip Erdoğan et son conseiller, Ahmet Davutoğlu, ont mis progressivement en avant un nouvel ordre du jour, moyenoriental et panislamique, correspondant davantage à leurs propres aspirations identitaires. Pour opérer ce tournant néanmoins risqué vis-à-vis de l'opinion publique, ils ont cherché à encourager et à structurer l'intérêt intellectuel diffus ${ }^{25}$ existant jusqu'alors pour le Moyen-Orient, et tenté de le convertir en une campagne de légitimation académique et médiatique. Cette stratégie est passée par la mise en place de relais entre le gouvernement, les experts individuels et les organisations qui produisaient ou diffusaient du savoir dans les champs universitaire, médiatique et diplomatique, en Turquie et à l'étranger. Ce sont des organismes de droit privé ${ }^{26}$ qui, en Turquie, se désignent du nom de düşünce kuruluşu - établissement de réflexion - ou think tank qui ont occupé cette position intermédiaire. Les deux premiers think tanks turcs, le Foreign Policy Council (Dış Politika Konseyi, DPK) et la Fondation pour les études sur la société, l'économie et le politique (Toplum, Ekonomi Siyaset Etüt Vakfi, TESEV) avaient été respectivement créés en 1973 et $1994^{27}$. Cependant, c'est à partir de la fin des années 1990 et du début des années 2000 que ces structures sont apparues par vagues successives ${ }^{28}$. L'une des plus puissantes de la seconde génération est le Centre de recherche stratégique sur l'Eurasie (Avrazya Stratejik Araştırma Merkezi, ASAM), créé en 1999 par l'universitaire et militant ultranationaliste Ümit Özdağ du parti ultranationaliste MHP (Milli Halk Partisi, Parti national du Peuple), qui a réuni une équipe de jeunes analystes, généralement non titulaires du doctorat, dont les recherches

${ }^{25}$ Et sans commune mesure avec l'européanisme ancien mais aussi décuplé par la perspective de l'adhésion à l'Union européenne. Lauren M. McLaren, «Turkey's Eventual Membership of the EU: Turkish Elite Perspectives on the Issue », Journal of Common Market Studies, 38 (1), 2000, p. 117- 129.

${ }^{26}$ Association (dernek) ou fondation (vakıf) suivant les cas.

${ }^{27}$ Hasan Kanbolat, «Think Tanks Are no Stepping Stones in Turkey, Sayfi Taşhan », Today’s Zaman, 22 décembre 2013.

${ }^{28}$ Hasan Kanbolat, Hasan A. Karasar, Türkiye'de Stratejik Düşünce Kültürü ve Sratejik Araştırma Merkezleri: Başlangıcından Bugüne Türk Düşünce Kuruluşları (La culture de la réflexion stratégique en Turquie et les centres de recherche stratégique), Ankara, Nobel Yayın Dağıtım, 2009, p. 12-15. 
étaient pilotées par la Fondation Avrazya-Bir, propriété de l'industriel Murat Ülker et dont le directeur n'était autre que Davutoğlu. Avec ASAM, les modalités des relations entre gouvernement, industriels et think tanks ont été établies pour deux décennies. En effet, les think tanks sont autant les producteurs plus ou moins sur commande d'un savoir gouvernemental que les réceptacles des gages financiers d'allégeance au pouvoir politique de certaines grandes entreprises. ASAM était ainsi financé par un entrepreneur qui, parce qu'il était soupçonné d'affinités islamo-conservatrices, devait tempérer son image en subventionnant une organisation dont les travaux - publications, conférences - avaient l'approbation de l'état-major et des responsables politiques nationalistes ${ }^{29}$. ASAM n'est pas le seul des think tanks turcs du début du millénaire à illustrer ce dispositif triangulaire entre décideurs politiques, élites économiques et producteurs de savoirs. Il semble même que la première partie de la décennie 2000 se soit caractérisée par une diversification croissante de ces organismes, y compris sur le plan idéologique. Ainsi, le Centre de recherche stratégique turc (Türk Stratejik Araştırma Merkezi, TÜRKSAM), petite structure nationaliste qui bénéficie des subsides publics et d'un soutien financier épisodique de la part de l'industrie turque de la Défense ${ }^{30}$, est apparue en 2004 au croisement du parti MHP, de foyers d'officiers en retraite et d'universités ankariotes. En 2005 a été également créée l'Organisation pour la recherche stratégique internationale (Uluslararas1 Stratejik Araştırma Kurulu, USAK), organisme réputé proche de la néoconfrérie de Fethullah Gülen mais aussi du futur président Abdullah Gül, et qui mettait en avant des personnalités et valeurs conservatrices sur le plan social mais libérales dans les domaines politiques économiques ${ }^{31}$.

Dans la seconde moitié des années 2000, une nouvelle vague de think tanks, pour la plupart proches des orientations politiques de l'AKP et dont certains des cadres étaient des transfuges des organisations de la vague précédente, a déferlé dans le paysage de l'expertise moyenorientale. Parmi ces nouveaux venus, deux think tanks détonnaient: la Fondation pour les Recherches sur la politique, l'économie et la société (Siyaset, Ekonomi, Toplum Araştırmalar Vakfi, SETA) et le Centre de recherches stratégiques sur le Moyen-Orient (Ortadoğu Stratejik Araştırmalar Merkezi, ORSAM), créé en 2008 par Hasan Kanbolat qui avait emmené avec lui quelques éléments d'ASAM. Par ses moyens financiers et son équipe, SETA était le plus important des think tanks. Ses dirigeants et sponsors entretenaient des rapports étroits avec le parti AKP et le gouvernement. Quant à ORSAM, il disposait de moyens financiers plus limités

\footnotetext{
${ }^{29}$ Ibid., p. 54.

${ }^{30}$ Entretien avec le vice-amiral Celalettin Yavuz, Ankara, avril 2013.

${ }^{31}$ Son président a été pendant longtemps Özdem Sanberk, ancien diplomate francophone, fils d'officier ottoman et ancien élève du lycée de Galatasaray.
} 
mais trouvait des appuis au sein du ministère des Affaires étrangères et parmi les élites de la minorité turkmène immigrées en Turquie tant en provenance d'Irak que de Syrie. En dépit de ses effectifs modestes, l'importance de cet organisme officiellement indépendant s'est pleinement révélée à partir de 2014, lorsque son fondateur a été limogé par le ministre des Affaires étrangères Davutoğlu qui l'a remplacé par Şaban Kardaş, un jeune enseignant-chercheur de 1'Université de l'Union des chambres de commerce et Bourses (Türk Odalar ve Bursalar Birliği, TOBB, Ankara), plus proche du ministre des Affaires étrangères. Cette décision politique appliquée à un organisme prétendant appartenir à la «société civile » annihilait l'indépendance et l'impartialité qu'il tentait de construire depuis sa création et attestait l'importance stratégique que lui accordaient les autorités AKP.

Par ailleurs, si les sciences politiques et administratives ont pu être qualifiées de sciences camérales ou « de gouvernement » et si la Turquie a une tradition ancienne de soumission de ces disciplines aux détenteurs du pouvoir politique ${ }^{32}$, Davutoğlu a clairement exprimé son désir que des centres de recherche soient créés afin d'éclairer la politique extérieure de la Turquie au Moyen-Orient ${ }^{33}$. Dès 1984 , estimant que l'enseignement qu'il recevait dans la prestigieuse Bosphorus (Boğaziçi Üniversitesi) ${ }^{34}$ manquait de références au monde musulman, il avait créé avec des camarades la Fondation Bilim ve Sanat Vakfi, qui s'est développée à la fin des années 1990 en diffusant des savoirs au croisement de l'histoire et des sciences religieuses dans le but de réhabiliter l'héritage ottoman dans une Turquie où les institutions républicaines et l'armée imposaient une amnésie collective sur cette période. Cette première initiative lui a servi à mettre en réseau une partie des intellectuels conservateurs musulmans et à rapprocher maîtres et disciples hors des murs de l'université, sur la base d'une communauté de vue conservatrice et islamique.

La nomination de Davutoğlu au poste de ministre des Affaires étrangères en 2009 a officialisé son ascendant sur l'appareil diplomatique. Elle lui a permis de veiller à ce que sa vision des savoirs de gouvernement se traduise par la consolidation, via la circulation de ses concepts et slogans - «zéro problème avec les voisins », « diplomatie multidirectionnelle », «diplomatie rythmique »-d'un dispositif institutionnel et discursif dont les chevilles ouvrières étaient SETA, ORSAM et le Centre de recherches stratégiques (Stratejik Araştırmalar Merkezi, SAM), lequel, relevant du ministère des Affaires étrangères, collaborait et échangeait du personnel avec les deux autres think tanks.

\footnotetext{
${ }^{32}$ E. Boğaç, I. Turan, «The Development of Political Science in Turkey », art. cité, p. 4.

${ }^{33}$ Ahmet Davutoğlu, Stratejik Derinlik. Türkiye'nin Uluslararası konumu (Profondeur stratégique. La position internationale de la Turquie), Istanbul, Istanbul İstanbul ? Küre Yayınları, 2001, p. 453.

${ }^{34}$ Anciennement Robert's College, Université américaine d'Istanbul.
} 
Mise en place d'un dispositif de production de savoirs de gouvernement dans l'interstice entre champ académique, domaine médiatique et appareil politico-administratif

Le choix du terme «dispositif»n'est pas neutre : dans le sens et la définition qu'en propose Foucault $^{35}$, il permet de restituer la cohérence d'un entrelacs de formes organisationnelles et discursives dans lequel se mêlent savoirs et pouvoir.

La multiplication et la diversification idéologique des «établissements de pensée stratégique » (stratejik düşünce kuruluşlart) dans la seconde moitié des années 2000 est un indicateur parmi d'autres de la perte de contrôle de l'état-major sur la politique extérieure. Le corrélat de cette éviction de l'armée est la revendication d'indépendance de ces organismes, revendication qui tient notamment à l'identité et aux trajectoires individuelles de leurs cadres. SETA, ORSAM, TEPAV (Toplumsal, Ekonomik ve Politik Araştırma Vakfı, Fondation pour les recherches sociétales, économiques et politiques) ou USAK ont été créés par de jeunes titulaires turcs de doctorats américains ou britanniques qui avaient souvent travaillé pour des organisations similaires durant leur séjour à l'étranger ${ }^{36}$. Pour constituer leurs équipes, les think tanks turcs se sont orientés vers plusieurs bassins de recrutement et quelques incubateurs spécifiques. Les chercheurs juniors de ces organismes avaient le plus souvent un master en sciences sociales et/ou en relations internationales des universités anglophones turques: la Middle East Technical (Ankara), Bilkent (Ankara) ou İstanbul Şehir ${ }^{37}$. Les diplômés de la très élitiste Bosphorus étaient en revanche plus rares.

Les cadres et collaborateurs extérieurs des think tanks, lorsqu'il s'agissait d'universitaires, étaient en général des docteurs en science politique (mention relations internationales) sortis d'universités anglo-saxonnes. En marge ou en complément de la filière internationale de recrutement, il existait ce que l'on pourrait appeler un «incubateur dédié », à savoir des établissements turcs fournissant à leurs étudiants les savoirs et l'insertion dans les réseaux leur permettant de devenir chercheur en think tank à l'issue de leurs études. C'est le cas de la

\footnotetext{
${ }^{35}$ Michel Foucault, Sécurité, territoire, population (1977-1978), Paris, Gallimard, 2004 ; Gilles Deleuze, «Qu'est-ce qu'un dispositif ? », dans Michel Foucault philosophe. Rencontre internationale, Paris, 9, 10, 11 janvier 1988, collectif, Paris, Le Seuil, 1989, p. 185- 195.

${ }^{36}$ La liste est longue, mais citons parmi d'autres : Talip Küçükçan (SETA), doctorat en anthropologie de Warwkick University ; Ufuk Ulutaş (SETA), master en science politique à l'Ohio State University ; Sedat Laçiner (USAK), doctorat en science politique de King's College (University of London) ; Şaban Kardaş (Université TOBB puis ORSAM), doctorat en science politique de Utah University.

${ }^{37}$ Fondée par Davutoğlu en 2008. Elle dépend de la fondation Bilim ve Sanat Vakfi.
} 
Fondation Bilim ve Sanat Vakfı (Fondation pour le savoir et les arts) de Davutoğlu, qui ne délivre pas de diplômes mais propose un parcours pédagogique sanctionné par des examens et bénéficie d'une reconnaissance en tant que complément extra-universitaire ès-islamologie et histoire de l'islam aux yeux des cadres de SETA ou de certains établissements universitaires marqués par leur proximité idéologique ou réticulaire avec le gouvernement, voire avec SETA ${ }^{38}$. L'Université de Sakarya, avec son Centre de recherche sur le Moyen-Orient (Ortadoğu Merkezi, ORMER), et İstanbul Şehir ont été également des incubateurs privilégiés dont certains diplômés et jeunes enseignants-chercheurs ont circulé intensément entre le Bilim ve Sanat Vakfi, les centres universitaires de recherche et les think tanks, entre 2008 et 2015. Ces organismes s'appuyaient aussi et surtout sur des collaborations avec des personnes et institutions extérieures. Leur capacité à capter, voire à coopter des ressources humaines dont ils ne sont pas toujours l'employeur principal est un autre indicateur de leur insertion tant dans le champ académique que dans les champs politico-administratifs des Affaires étrangères et de l'Enseignement-Recherche. De manière plus générale, la politique moyen-orientale menée à partir de $2006^{39}$ et sa promotion polymorphe par Davutoğlu et son aréopage académico-politique ont créé un effet de mode qui a touché les universités, les fondations culturelles enseignant l'arabe et l'histoire de la civilisation islamique, et ces think tanks qui assuraient l'articulation entre milieu académique, appareil politico-administratif et financeurs privés ${ }^{40}$. De cette mode a résulté un accroissement de l'offre de formation - sciences sociales et langues - sur le Moyen-Orient, ainsi qu'une multiplication des instances de recherche dédiées, publiques comme privées ${ }^{41}$. Cette hausse de l'offre a rencontré une hausse de la demande puisque la génération d'étudiants qui fréquentaient les facultés de sciences sociales à la fin des années 2000 aspiraient au renouvellement des contenus de leurs enseignements dans le sens d'une ouverture sur le monde - pas seulement occidental et d'une reformulation de l'identité turque ${ }^{42}$. Ceux qui étaient issus de milieux conservateurs ont joué un rôle prépondérant dans la montée en puissance des études moyen-orientales en Turquie.

\footnotetext{
${ }^{38}$ Entretien avec Tuba Kor, Fondation Bilim ve Sanat, Istanbul, décembre 2012 ; entretien avec Hatem Ete, SETA, Ankara, janvier 2013 ; entretien avec Ertan Efeğil, Université de Sakarya, Istanbul, février 2014.

${ }^{39}$ Voir note $\mathrm{n}^{\circ} 1$, et Dorothée Schmid (dir.). La Turquie au Moyen-Orient : le retour d'une puissance régionale?, Paris, CNRS Éditions, 2011.

${ }^{40}$ Financeurs qui cherchaient, comme dans le cas de celui d'ASAM, à prouver leur allégeance au gouvernement AKP : Ülker Grubu, Çalık Grubu, Turkuaz Medya Grubu...

${ }^{41}$ Les personnes que j'ai rencontrées ont souligné la nouveauté des cours sur le Moyen-Orient dans leurs universités respectives. Entretien avec Meliha Benli Altunışık, Université METU, Ankara, janvier 2013 ; entretien avec Beril Dedeoğlu, Université de Galatasaray, janvier 2014.

${ }^{42}$ Entretiens avec Cengiz Tomar (directeur adjoint) et Ahmet Uysal (maître de conférences en science politique), Institut pour les recherches sur le Moyen-Orient de l'Université de Marmara, respectivement en décembre 2012 et janvier 2014.
} 
Les universités publiques et privées ont commencé à proposer des cours de «Middle East Politics », voire des cours d'arabe ${ }^{43}$, certaines, même, des masters d'affaires internationales centrés sur le Moyen-Orient comme l'université de Sakarya, Istanbul Şehir (master préparant à la carrière diplomatique) ou Bilkent. Dans la seconde moitié des années 2000, chacune a créé son propre centre de recherche dédié au Moyen-Orient, comme les Universités de Bolu, en Anatolie centrale, et d'Elazı̆̆ en Anatolie orientale, ou l'a renforcé s'il existait déjà comme l'Université de Sakarya qui a ouvert ORMER en 2011, sur la base d'une structure préexistante, Travaux sur le Moyen-Orient (Ortadoğu Çalışmalar), ou l'Université de Marmara qui avait un Institut de recherche (et d'enseignement) sur le Moyen-Orient depuis 1996. Autant de futurs foyers de recrutement pour les think tanks, la presse et le ministère des Affaires étrangères.

De l'internationalité à la transdisciplinarité : la dimension générationnelle des trajectoires d'experts

Dans mes travaux précédents, l'analyse du champ de l'expertise moyen-orientale turque dans les années 2000 et 2010 passait par l'étude des positions occupées par les acteurs individuels et des parcours qui les y avaient amenés. Deux options théoriques semblaient alors possibles : celle qui fait usage de la notion de «trajectoire », elle-même liée aux concepts bourdieusiens d'habitus et de champ, utile pour mettre en évidence les passages des acteurs entre espaces sociaux; et celle développée par Howard Becker ${ }^{44}$ et plus tard par la sociologie politique française ${ }^{45}$ qui, en recourant à la notion de «carrière », permet d'analyser à la fois les motifs personnels d'engagement et les positions successives. Ne souhaitant pas être enfermé dans une étude du militantisme qui ne convenait pas à l'ensemble des parcours recensés, j'ai opté pour l'utilisation du terme de trajectoire. Sur les deux générations qui, en 2010, avaient, pour l'une, entre 40 ans et 50 ans, pour l'autre, entre 20 ans et 40 ans $^{46}$, j'ai dénombré cinq « parcours-types ${ }^{47}$.

- Parcours 1 : génération 40 ans-50 ans : études undergraduate de relations internationales et/ou de science politique en anglais dans une université turque prestigieuse (Boğaziçi,

\footnotetext{
${ }^{43}$ Entretien avec N. Mert cité ; entretien avec Muzaffer Şenel (Université İstanbul Şehir), Istanbul, novembre 2012 ; entretien avec Gencer Özcan (Université Bilgi), Istanbul, octobre 2012.

${ }^{44}$ Howard Becker, Outsiders. Studies in the Sociology of Deviance, New York, Free Press, 1963.

${ }^{45}$ Olivier Fillieule, Nonna Mayer, «Devenirs militants. Introduction», Revue française de science politique, 51 (1-2), 2001, p. 23.

${ }^{46}$ Les seconds ayant été les étudiants des premiers mais pas toujours car beaucoup ont effectué leur cursus postgraduate à l'étranger.

${ }^{47}$ Cette typologie est tirée de l'analyse prosopographique mentionnée plus haut.
} 
METU) $\rightarrow$ doctorat dans la même discipline aux États-Unis ou au Royaume-Uni dans une université prestigieuse (Oxford, Harvard, Columbia) $\rightarrow$ enseignement-recherche dans une université anglophone prestigieuse en Turquie (intérêt professionnel pour le MoyenOrient à partir du doctorat)

- Parcours 2: génération 40 ans-50 ans : licence et maîtrise de relations internationales et/ou de science politique en turc $\rightarrow$ doctorat dans la même discipline aux États-Unis ou au Royaume-Uni dans une université non prestigieuse (Ohio State, Utah State, George Mason) $\rightarrow$ enseignement-recherche dans une université turque de rang moyen (Marmara, Sakarya, Bolu)

- Parcours 3: génération 40 ans-50 ans : lycée İmam hatıp ${ }^{48}$ puis licence de théologie islamique $\rightarrow$ licence $\left(2^{\mathrm{e}}\right)$ et master de science politique ou de sociologie $\rightarrow$ doctorat de science politique ou de sociologie dans une université américaine ou britannique non prestigieuse (les mêmes que pour le parcours 2) $\rightarrow$ enseignement-recherche dans une université turque de rang moyen (les mêmes que pour le parcours 2)

- Parcours 4 : génération 20 ans-40 ans : licence et maîtrise de relations internationales et/ou de science politique en turc dans une université prestigieuse ou non (METU, Ankara, Istanbul, Sakarya, Marmara, Bolu) $\rightarrow$ doctorat en cours ou inachevé dans une université turque $\rightarrow$ chargé d'enseignement à l'université durant la réalisation du doctorat

- Parcours 5 : Les deux générations 20 ans-40 ans et 40 ans-50 ans : licence de sciences sociales $\rightarrow$ expérience militante dans un parti de gauche radicale ou dans une organisation humanitaire islamo-conservatrice $\rightarrow$ emplois de journalistes (presse écrite, télévision, envoyés spéciaux/reporters/documentaristes) $\rightarrow$ rédacteur en chef/chef de programme d'une chaîne de télévision/essayiste

Ces trajectoires moyen-orientalistes vont de pair avec le multipositionnement ${ }^{49}$ des acteurs individuels entre plusieurs institutions et secteurs d'activité : enseignement supérieur et administration au sein de l'université, participation au comité scientifique d'une revue, positions de prestige dans un think tank et éventuellement sollicitations occasionnelles en tant que conseiller du prince ou expert médiatique, haute administration (Affaires étrangères). Les positions occupées se renforcent mutuellement tandis que le niveau d'interconnaissance des

\footnotetext{
${ }^{48}$ École secondaire d'imams prédicateurs dont les contenus pédagogiques sont centrés sur l'enseignement religieux.

${ }^{49}$ Luc Boltanski, «L'espace positionnel : multiplicité des positions institutionnelles et habitus de classe », Revue française de sociologie, 14 (1), 1973, p. 3- 26.
} 
acteurs et les capitaux spécifiques mobilisés apparaissent clairement. Les parcours types que j’ai identifiés peuvent ainsi être corrélés à certaines combinaisons de positions entre divers secteurs d'activité, qui assurent la porosité transsectorielle du champ d'expertise. La nature du cursus universitaire (discipline, lieu des études, en Turquie ou à l'étranger) semble plus déterminante dans la phase d'insertion professionnelle que l'affiliation politique progouvernementale qui, elle, a le plus souvent un effet sur le rythme de l'ascension professionnelle et le cumul des positions. L'expertise du Moyen-Orient est aussi un facteur de fluidité entre mondes professionnels dans la mesure où elle permet à certains acteurs d'occuper des positions pour lesquelles ils ne disposent pas a priori des capitaux symboliques requis. C'est le cas de certains journalistes vedettes vis-àvis du milieu universitaire et des think tanks, mais aussi des théologiens vis-à-vis des sciences sociales, ces experts du Moyen Orient «par appartenance », puisque l'islam est communément considéré comme une religion moyen-orientale, devenant alors des «experts par profession», pour reprendre la distinction opérée par H. Becker ${ }^{50}$.

Ces cumuls individuels de fonctions s'expliquent par la concentration des positions de pouvoir au bénéfice de quelques acteurs se connaissant tous. Il en résulte une certaine cohérence du champ expert qui aboutit à l'uniformisation des thématiques de recherche et sujets médiatiques légitimes en ce qui concerne le Moyen-Orient et la politique moyen-orientale du gouvernement. En résulte également une concentration des financements sur certains acteurs institutionnels, et ce d'autant plus que les think tanks très proches des cercles ministériels, comme SETA et ORSAM, sont non seulement éligibles aux bourses publiques délivrées par le Conseil turc pour la recherche scientifique et technologique ${ }^{51}$ mais manifestement plus performants que les centres de recherche publics quand il s'agit d'obtenir des financements internationaux ${ }^{52}$.

Quelques exemples concrets de ces multipositionnements permettent d'illustrer cette monopolisation. Entre 2005 et 2008, İbrahim Kalın, universitaire et conseiller de Davutoğlu, fonda SETA à Ankara avec une poignée de jeunes docteurs fraîchement revenus, comme lui, des États-Unis. Parmi les premiers cadres de recherche recrutés et les invités aux premiers événements organisés, on trouve d'emblée les partisans et/ou anciens disciples de Davutoğlu, notamment ceux qui venaient de l'Université d'İstanbul Şehir, récemment ouverte, du Bilim ve Sanat Vakfi et/ou du département anglophone de relations internationales de l'Université de Marmara où Davutoğlu enseignait au moment où il a été sollicité par le gouvernement Gül. En

\footnotetext{
${ }^{50}$ H. Becker, Outsiders. Studies in the Sociology of Deviance, op. cit.

${ }^{51}$ Entretien avec Hasan Kösebalaban, Université İstanbul Şehir, décembre 2012 ; entretien avec Mensur Akgün, TESEV, Istanbul, novembre 2012.

${ }^{52}$ Entretien avec H. Kanbolat, ORSAM, Ankara, avril 2013 : entretien avec Sabiha Gündoğar, TESEV, Istanbul, janvier 2014.
} 
2012, les dirigeants de SETA qui en constituaient le trio de tête ${ }^{53}$ étaient également conseillers du ministre des Affaires étrangères et/ou membres du comité consultatif du SAM. İbrahim Kalın resta peu de temps à SETA. En 2009, il intégra le cabinet Erdoğan, au sein duquel il devint conseiller du Premier ministre pour la politique étrangère, poste occupé jusque-là par Davutoğlu. On note aussi des profils plus singuliers comme celui de Talip Küçükçan, théologien formé à la School of Oriental and African Studies à Londres. Devenu professeur de sociologie à l'Université de Marmara, il fut également directeur de l'Institut pour les recherches sur le Moyen-Orient de cette université (2011-2014) et directeur des études de politique étrangères à SETA (2009-2013). Outre un programme de politique étrangère sur la chaîne nationale TRT, il fut aussi rédacteur en chef de la revue à comité de lecture Insight Turkey, publiée par SETA. À partir de 2010, il fut de surcroît conseiller auprès du président du Conseil de l'enseignement supérieur. Il quitta le milieu académique en 2015 et fut élu au Parlement en tant que député d'Adana.

SETA est donc à la fois un lieu de sociabilité, un nœud réticulaire et une antichambre du pouvoir technocratique. Outre leurs activités académiques et technocratiques, ces «janissaires intellectuels » du parti AKP ont une visibilité médiatique puisqu'ils animent sur TRT des programmes destinés, sous couvert de débat ou de pédagogie, à diffuser la grille de lecture de la politique internationale du gouvernement.

Si l'on considère ce nœud depuis le pôle universitaire, il s'avère que nombre d'enseignantschercheurs de relations internationales à İstanbul Şehir, également enseignants bénévoles à la fondation Bilim ve Sanat, participent aux activités de SETA, voire y occupent des fonctions d'encadrement. SETA puise également dans d'autres viviers que les universités par lesquelles est passé Davutoğlu : l'Université de Sakarya et son centre dédié, ORMER, lui fournissent plusieurs spécialistes pour ses ateliers et la revue Insight Turkey.

Par ailleurs, certains enseignants-chercheurs de relations internationales et de science politique de l'Université Middle East Technical d'Ankara figurent dans les comités scientifiques de SETA, ORSAM, USAK et TEPAV. Le positionnement de ces acteurs est toutefois moins concentré sur SETA et ses partenaires (İstanbul Şehir, Bilim ve Sanat, Sakarya) et concerne moins des positions d'encadrement que de conseil et de cautionnement scientifique. S'ils ont une stratégie de positions multiples, ils restent centrés sur le champ académique (université, YÖK, revues). Leur soutien est d'autant plus précieux qu'il est auréolé d'indépendance : ils ne peuvent en effet être qualifiés d' « intellectuels organiques » comme ceux qui dirigent SETA et qui, en

\footnotetext{
${ }^{53}$ Le président, le directeur des recherches de politique intérieure et le directeur des recherches de politique étrangère.
} 
dépit de leurs titres multiples et de leur mainmise sur les financements publics, ont un crédit expert plus limité hors du cercle partisan de l'AKP.

La dynamique réticulaire s'étend à l'international. Le relevé des participations étrangères aux conférences, ateliers et numéros de revues des think tanks turcs consacrés au Moyen-Orient entre 2008 et 2015 fait apparaître la prédominance des universités anglo-saxonnes et notamment de celles ou les experts turcs du Moyen-Orient ont effectué leur doctorat. En outre, pour renforcer leur crédibilité académique, les think tanks comme SETA et USAK mettent en avant le financement obtenu de la part d'organisations comme la Carnegie Endowment for Peace, le German Marshall Fund. Cependant, il faut relativiser le phénomène de monopolisation des ressources puisque ces flux de financement transnationaux profitent également à d'autres think tanks. L'Open Society Foundation a ainsi soutenu la création et les projets de recherche de TESEV, organisation stambouliote parmi les plus anciennes du secteur qui a également reçu des fonds de la Carnegie et du German Marshall Fund.

Janissaires du savoir : consolidation d'une communauté épistémique au cœur du champ

Le multipositionnement d'une constellation d'acteurs et leur présence dans un nombre relativement restreint d'organisations tendent à délimiter les contours d'un champ expert et à assurer la cohérence de ce qui s'y produit tant sur la forme que sur le fond. La cohésion, quant à elle, semble tenir à l'existence d'un groupe plus soudé au cœur même de ce champ. C'est ce que j'ai choisi d'appeler la «communauté épistémique davoutolienne » car sa structure comme ses références renvoient à la personne et/ou à l'œuvre de Davutoğlu. Pour caractériser ce groupe d'intellectuels organiques de l'AKP, je me suis appuyé sur les spécificités dégagées par Peter Haas $^{54}$ et les ai rapportées tant au contenu des entretiens menés en Turquie entre 2012 et 2015 qu'à la prosopographie retraçant le parcours de 140 individus s'étant signalés par leur production de savoirs sur le Moyen-Orient en Turquie entre 1998 et 2015. L'intérêt de ce concept est qu'il met l'accent sur les rapports entre acteurs académiques et administrations d'État. Les membres d'une communauté épistémique partagent une même grille de lecture du monde - causal beliefs -, un rapport consensuel au savoir et une même conception de leur relation avec les acteurs politico-administratifs.

\footnotetext{
${ }^{54}$ P. M. Haas, Knowledge, Power, and International Policy Coordination, op. cit., p. 3.
} 
Ceux de la communauté épistémique davoutolienne (CED) relèvent essentiellement des profils 2, 3 et 4 définis plus haut. Les plus éminents ( 2 et 3 ) ont effectué leur doctorat aux États-Unis, souvent dans les mêmes universités. La détention d'un doctorat étranger est un facteur de hiérarchisation comme dans l'ensemble du système universitaire turc. Tous sont issus d'un milieu familial conservateur dans le sens religieux et ont suivi un cursus extrascolaire de familiarisation à l'islam qui s'est quelquefois prolongé jusqu'à l'université. C'est notamment le rôle joué par la fondation Bilim ve Sanat pour ceux qui ont étudié à Istanbul.

La consolidation de cette communauté s'est effectuée dans la seconde moitié des années 2000, avec la multiplication des think tanks turcs. Ses membres étaient actifs simultanément à l'université, dans l'administration et dans les médias : ils ont accru leur influence collective sur la production et la diffusion des savoirs en lien avec la politique moyen-orientale du gouvernement et veillé à ce que le dispositif - énoncés, organisations - soit cohérent avec les décisions politiques de l'AKP.

\section{Le champ des experts turcs du Moyen-Orient à maturité (2012) ${ }^{55}$}



\footnotetext{
${ }^{55}$ Le schéma fait état de polarités idéologiques sur lesquelles je ne m'attarde pas ici. Il s'agissait de restituer l'influence de l'idéologie et/ou des affiliations partisanes des acteurs sur leur positionnement sachant que, hormis les proches de l'AKP et des ultra-nationalistes, il pouvait ne s'agir que d'une lointaine affinité idéologique. C'est notamment le cas de la polarité gauche radicale à laquelle ont pu appartenir certains experts du Moyen-Orient dans leur jeunesse avant de s'en éloigner jusqu'à devenir conseillers du prince.
} 
Un dispositif fragilisé par les insurrections arabes

Les ambitions hégémoniques de cette communauté se sont révélées au lendemain des insurrections libyenne et égyptienne, tandis qu'apparaissaient les premières divergences significatives d'interprétation ou de recommandation avec des experts idéologiquement proches des ultranationalistes et de la gauche modérée ou radicale. Jusque-là, l'hétéronomie financière du champ et sa dépendance partielle vis-à-vis des subventions publiques ${ }^{56}$ ne semblaient pas avoir eu d'effet mécanique direct sur ses activités ${ }^{57}$. Le seul fait de produire et de diffuser des savoirs portant sur la politique moyen-orientale du gouvernement ou sur la région allait dans le sens de la politique gouvernementale dont l'orientation moyen-orientale était en soi un défi à l'ordre antérieur caractérisé par une politique étrangère atlantiste et dominée par l'armée. Tant que la politique moyen-orientale de l'AKP a suivi une courbe ascendante, les différends importaient peu. Or la chute en série des régimes autoritaires arabes avec lesquels Ankara avait traité a obligé le gouvernement à diverses contorsions qui ont contredit le récit d'une politique pragmatique et fragilisé le relatif consensus expert construit au cours des années 2000.

L'hétéronomie financière a commencé à avoir des effets mécaniques sur la production et l'implication des acteurs à partir de 2012 et de la campagne de promotion du modèle turc au Proche-Orient et en Afrique du Nord, entreprise dont le champ expert a été l'un des acteurs centraux $^{58}$. Il semble en effet que le choix des thématiques et des contributeurs ait opéré un virage qui annonçait une restriction progressive du champ expert à la communauté épistémique, autrement dit la disparition d'un champ animé d'un semblant d'autonomie en faveur d'une légion d'intellectuels organiques dominant la production analytique non seulement sur la politique extérieure mais aussi sur l'ensemble des savoirs produits en Turquie concernant le

\footnotetext{
${ }^{56}$ Peu traitée ici, cette dépendance financière est difficilement démontrable par des chiffres puisque les quelques rapports d'activité publiés par les think tanks turcs ne livrent pas de comptabilités détaillées. Elle est toutefois mentionnée de manière récurrente tant par des chercheurs au sein de ces organismes que par des acteurs plus indépendants. Entretien avec Kerim Balc1, Turkish Review, Istanbul, février 2012; entretien avec Celalettin Yavuz, TÜKSAM, Ankara, janvier 2013 ; entretien avec Oytun Orhan, ORSAM, Ankara, janvier 2014.

${ }^{57} \mathrm{Ce}$ constat repose sur une analyse systématique du contenu des revues dites «académiques » publiées par les principaux think tanks et quelques universités turques entre 2008 et 2015 : Insight Turkey (SETA), Ortadoğu Etütleri (ORSAM), Akademik Ortadoğu (Université d'Ankara), Perceptions (SAM, ministère des Affaires étrangères).

${ }^{58}$ De nombreux articles et ouvrages sont alors parus en Turquie, dont certains donnaient dans la critique frontale de la politique étrangère turque. Murat Aktaş, Nilüfer Göle, Cengiz Aktar, Abdullah Kıran, Cenap Çakmak, Fulya Şen, Orkhan Gafarlı (dir.), Arap Baharı. Ortadoğu'da demokrasi arayışı ve Türkiye Modeli (Printemps arabes. Recherche de démocratie et modèle turc au Moyen-Orient), Ankara, Nobel Yayın Dağıtım, 2012.
} 
Moyen-Orient. Du milieu de l'année 2012 au milieu de l'année 2013, alors que le conflit syrien dégénérait en guerre civile et que le gouvernement de l'AKP perdait le bénéfice de ses bonnes relations avec ce voisin, les revues académiques des think tanks turcs mais aussi les symposiums qu'ils organisaient avaient pour thématique phare le «modèle turc ». C'est durant cette période que SETA a ouvert une succursale au Caire, dans le but de relayer la vision turque du MoyenOrient et d'entrer en contact avec les acteurs locaux ${ }^{59}$. Les affrontements en Égypte et en Tunisie s'étant apaisés, un nouvel ordre a semblé se mettre en place, non sans tensions, et une poignée d'enseignants-chercheurs et d'analystes, à ORSAM et USAK notamment, se sont rendus dans ces deux pays à des fins ethnographiques ${ }^{60}$. Lorsqu'ils effectuaient des voyages officiels, comme lors de la tournée triomphale dans la région en 2011, Erdoğan et Davutoğlu étaient non seulement accompagnés de journalistes et d'entrepreneurs mais aussi de ces «janissaires du savoir » qui gravitaient autour des think tanks turcs. Leur mission consistait à identifier et contacter les acteurs locaux issus des révolutions ${ }^{61}$.

Parallèlement, les élites turques exprimaient de plus en plus haut leur désaccord avec la politique syrienne du gouvernement, parfois accusée de confessionnalisation prosunnite, l'accueil de millions de réfugiés syriens en Turquie et la posture vindicative des autorités à l'égard des alliés occidentaux. L'acharnement judiciaire du gouvernement contre les médias, puis contre les membres supposés de la néoconfrérie guléniste, a conduit certains intellectuels, en particulier ceux qui étaient spécialisés dans la politique moyen-orientale, à se prononcer selon les trois options définies par Albert Hirschman : la sortie, l'opposition, la loyauté (exit, voice, loyalty) ${ }^{62}$. Parmi les individus et les institutions périphériques ou autonomes de la configuration experte qui s'était constituée au cours des dix années précédentes, beaucoup ont choisi de se recentrer sur l'enseignement tout en adoptant une posture critique modérée. Cette option n'a en réalité été possible que jusqu'à la «Pétition pour la paix » lancée en janvier $2016^{63}$, moment charnière à partir duquel même la critique modérée de la politique gouvernementale conduit à un licenciement des organisations vers lesquelles ces intellectuels s'étaient repliés (universités, organes médiatiques, think tanks). Quoiqu'il en soit, pour ces intellectuels, choisir l'exit signifiait

\footnotetext{
${ }^{59}$ Entretien avec Taha Özhan, directeur de SETA, Ankara, janvier 2013.

${ }^{60}$ Entretien avec Özlem Tür, Université METU, Ankara janvier 2013 ; entretien avec Meliha Benli Altunışı, Université METU, Ankara, janvier 2013.

${ }^{61}$ Entretien avec Burcu Gültekin, Ankara, janvier 2014.

${ }^{62}$ Albert O. Hirschman, Exit, Voice, and Loyalty: Responses to Decline in Firms, Organizations, and States, Cambridge, Harvard University Press, 1970.

${ }^{63}$ Pétition internationale ayant circulé dans le monde académique turc et à l'étranger pour réclamer la cessation des hostilités et la reprise des pourparlers de paix entre l'État turc et le PKK. Elle déclencha une offensive disciplinaire et judiciaire du gouvernement contre les universitaires signataires. Olivier Grojean, La révolution kurde. Le PKK et la fabrique d'une utopie, Paris, La Découverte, 2017.
} 
déjà renoncer aux publications dans les tribunes académiques qui avaient accompagné leur ascension professionnelle. La seconde option, choisie par plusieurs experts du Moyen-Orient anciennement militants de la gauche radicale, a consisté à critiquer durement les choix du gouvernement (voice). C'était une décision lourde de conséquences, notamment financières, car elle les exposait à des procédures judiciaires systématiques, surtout depuis le coup d'État manqué de juillet 2016 et l'instauration de l'état d'urgence qui s'était ensuivie. La troisième option, celle de la communauté épistémique et de quelques experts issus de la tendance ultranationaliste ayant rallié l'AKP, se caractérise par un zèle inédit dans la justification et la légitimation de la politique extérieure du gouvernement (loyalty), quels que soient ses errances et son manque de lisibilité, en Syrie comme en Égypte ${ }^{64}$. C'est en particulier le cas du personnel d'ORSAM, dont les directeurs successifs avaient été limogés entre 2014 et 2017 mais dont les principaux cadres sont restés en place.

Enfin, lorsque Davutoğlu a quitté la primature, l'allégeance a été transférée vers Erdoğan et ses partisans. En témoignent, d'une part, le fait que les cadres de SETA aient poursuivi leur carrière dans l'administration de la Présidence ou au ministère des Affaires étrangères, d'autre part, le fait que les partisans de la première heure d'Erdoğan ${ }^{65}$ aient commencé, à partir de 2016, à occuper des positions plus prestigieuses mais aussi plus dépendantes du pouvoir politicoadministratif (postes de professeurs, postes dans la haute administration universitaire, direction d'ORSAM...).

Après des décennies de tensions, la normalisation des relations turco-syriennes en 1998 a produit un engouement inédit pour le Proche-Orient arabe dans certains milieux intellectuels turcs, universitaires et médiatiques notamment. Le champ expert lié à la politique moyen-orientale de 1'AKP qui s'est mise en place à la fin des années 2000 est comparable à bien des égards à ce qui a pu être observé dans d'autres pays dans le sillage d'une politique d'État ou d'une action publique. De même, le caractère caméral de certaines disciplines scientifiques, leur soumission à un besoin politique de produire des savoirs de gouvernement sont une composante non originale

\footnotetext{
${ }^{64}$ Entretien avec Ufuk Ulutaş, directeur des études de politique étrangère à SETA, Ankara, décembre 2014.

${ }^{65}$ Lesquels ont souvent eu, à l'exception d'İbrahim Kalın, son porte-parole, un parcours académique plus « turc » qu'international et occupaient donc à la fin des années 2000 des positions plus limitées en nombre et en prestige que leurs collègues au cursus internationalisé.
} 
du phénomène étudié ici. La spécificité du cas turc repose peut-être sur la création et l'imbrication presque concomitantes d'un domaine d'étude scientifique et d'une communauté épistémique au sein de celui-ci. La brièveté de l'existence du champ d'études moyen-orientales turc, dix ans à peine, rapidement rattrapé par son défaut congénital, la dépendance vis-à-vis du gouvernement, incite à une interprétation plus générale. De fait, la fusion entre la dynamique experte -individus, organisation, fonctionnement - et le gouvernement avec lequel elle collaborait sur un mode consensuel annonçait sa disparition prochaine. Le politique n'instrumentalise la science et ne se montre bienveillant face à son effort impartial de décryptage de la réalité objective que lorsque leurs agendas coïncident. Si l'objectif de la science ne varie pas, l'agenda politique, lui, varie au gré des circonstances. Dès lors, à moyen et long terme, la « circulation des priorités » propre au politique n'offre aux experts que deux options : celle de rejoindre le politique en renonçant largement à ce qui leur a conféré leur crédit scientifique et à la liberté dont ils bénéficiaient à l'interface entre les deux champs; ou celle de s'éloigner du politique pour regagner leur secteur d'origine, renonçant cette fois aux bénéfices symboliques de la position d'expert. Par ailleurs, dans un pays où la conception camérale des sciences est historiquement défendue par l'autorité politique et où le monde académique est en partie structuré par des luttes idéologiques, les acteurs sont constamment sommés de se situer politiquement. Et ils le font, ne serait-ce que parce que leur position dans leur secteur d'appartenance en dépend en grande partie.

Jean-Baptiste Le Moulec est docteur en science politique (Aix-en-Provence, 2016) et vacataire. De 2017 à 2019, ses recherches ont porté sur le dispositif d'influence turc en Afrique du Nord. jb.lemoulec@gmail.com 